\title{
Educational note: Paradoxical collider effect in the analysis of non-communicable disease epidemiological data: a reproducible illustration and web application
}

\section{Miguel Angel Luque-Fernandez, Michael Schomaker, Daniel Redondo-Sanchez, Maria Jose Sanchez Perez, Anand Vaidya and Mireille E Schnitzer}

First published online: 10 December 2019, Int J Epidemiol 2019; Volume 48, Issue 2, April 2019, Pages 640-653.

doi: https://doi.org/10.1093/ije/dyy275

The corrigendum to this article published on 10 December 2019 (https://doi.org/10.1093/ije/dyz247) referred to corrections made to the code in Box 2. These corrections were in fact, incorrect, as was the sentence referring to Box 2. The correct text and Box 2 code is as follows (changes shown in bold).
"The true causal coefficient of the exposure A is 0.3 , and the coefficients for the association of the collider $\mathrm{C}$ with the exposure A and the outcome $\mathrm{Y}$ are $\mathbf{1 . 2}$ and 0.9 , respectively (Box 2).”

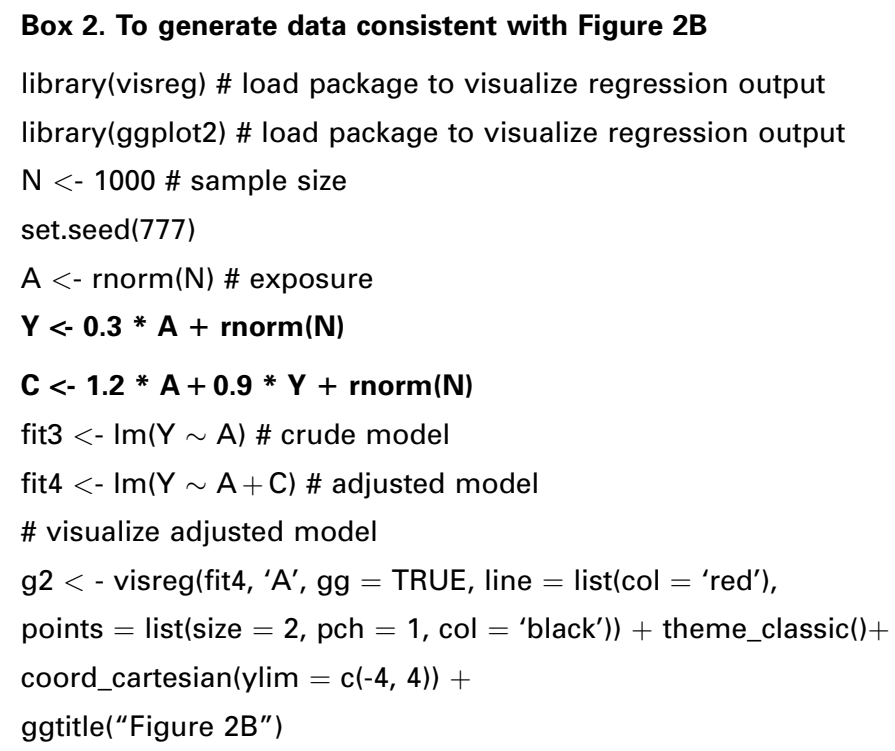

